\title{
From Decision Knowledge to E-Government Expert Systems: The Case of Income Taxation for Foreign Artists in Belgium
}

\author{
Faruk Hasića,*, Jan Vanthienen $^{1}$ \\ ${ }^{a}$ Research Centre for Information Systems Engineering (LIRIS), KU Leuven \\ Naamsestraat 69, 3000 Leuven, Belgium
}

\begin{abstract}
Since the introduction of the Decision Model and Notation (DMN), the standard has successfully been adopted in both industry and academia. However, no clear modelling guidelines can be found regarding the development of DMN decision models. For approaching this gap, this paper discusses modelling methodologies for the DMN standard, both at the decision requirements level as well as at the decision logic level. For that purpose, we capitalise on existing guidelines in related fields, such as decision table modelling, information systems engineering, and software engineering. We adapt, expand, and restructure the guidelines to confrom to DMN model development. Additionally, we provide a real-life case that was modelled using the suggested modelling strategies and consequently, we deploy the model as a government e-service for tax management. The real-life case is concerned with clarifying tax regulations for visiting performing artists in Belgium, and it was carried out in cooperation with oKo, a Belgian industry federation for the arts, and the Ministry of Culture. The decision model was built in the Avola tool and implemented as an e-service in order to demonstrate the suitability of the DMN standard for the deployment of expert system e-government services.
\end{abstract}

Keywords: Expert Systems, Decision Modelling, Decision Model and Notation, DMN, E-Government, E-Services.

\footnotetext{
* Corresponding author

Email address: faruk.hasic@kuleuven.be (Faruk Hasić)
} 


\section{Introduction}

Decision modelling has seen a surge in scientific literature, as illustrated by the vast body of recent work on Decision Model and Notation (DMN) (OMG, 2019; Ghlala et al., 2017; Mertens et al., 2017; Hasić et al., 2018b; Hasić \& Vanthienen, 2019; Hasić et al., 2018a; De Smedt et al., 2016; Santoro \& Baião, 2017; Hasić et al., 2017b; De Smedt et al., 2019; Deryck et al., 2018). Numerous tool developers already incorporated DMN modelling in their software, making the standard available for industry. DMN consists of two levels. Firstly, the decision requirement level in the form of a Decision Requirement Diagram (DRD) is used to portray the requirements of decisions and the dependencies between the different constructs in the decision model. Secondly, the decision logic level is used to specify the underlying decision logic, usually in the form of decision tables. The standard also provides boxed expressions and decision tables for the notation of the decision logic, as well as the FEEL expression language (Friendly Enough Expression Language). Every FEEL expression in a decision model has clearly defined execution semantics OMG (2019).

In DMN rectangles are used to depict decisions, corner-cut rectangles for business knowledge models, and ovals to represent data input. The arrows represent information requirements (from data or decisions). DMN is a declarative decision language. Hence, DMN provides no decision resolution mechanism, this is left to the invoking context. The same holds for the processing and storage of outputs and intermediate results, as this is a task of the invoking entity (e.g. a business process). A link can be made between a decision task in the process model and the actual decision model, as show in the example in Figure 1, where the decision model is invoked as a service by the process model, indicated by the dashed line. That way, the process activity Decide Eligibility can utilise the decision logic encapsulated in the DMN decision model on the left-hand side of the figure. Despite the adoption of the DMN standards in both industry and academia, a discussion on DMN modelling strategies is still absent in literature. Additionally, the use of DMN for the creation of e-services has not been demonstrated yet. In this paper, we shed a light upon DMN modelling strategies based on previous works and general concepts from process management and conceptual modelling. Additionally, we follow the proposed strategies to build a reallife complex decision model on the problem of tax regulations for visiting performing artists in Belgium. The model was built in the Avola decision management tool in cooperation with oKo, a Belgian industry federation for the arts, and the Ministry of Culture, and it was implemented as an 


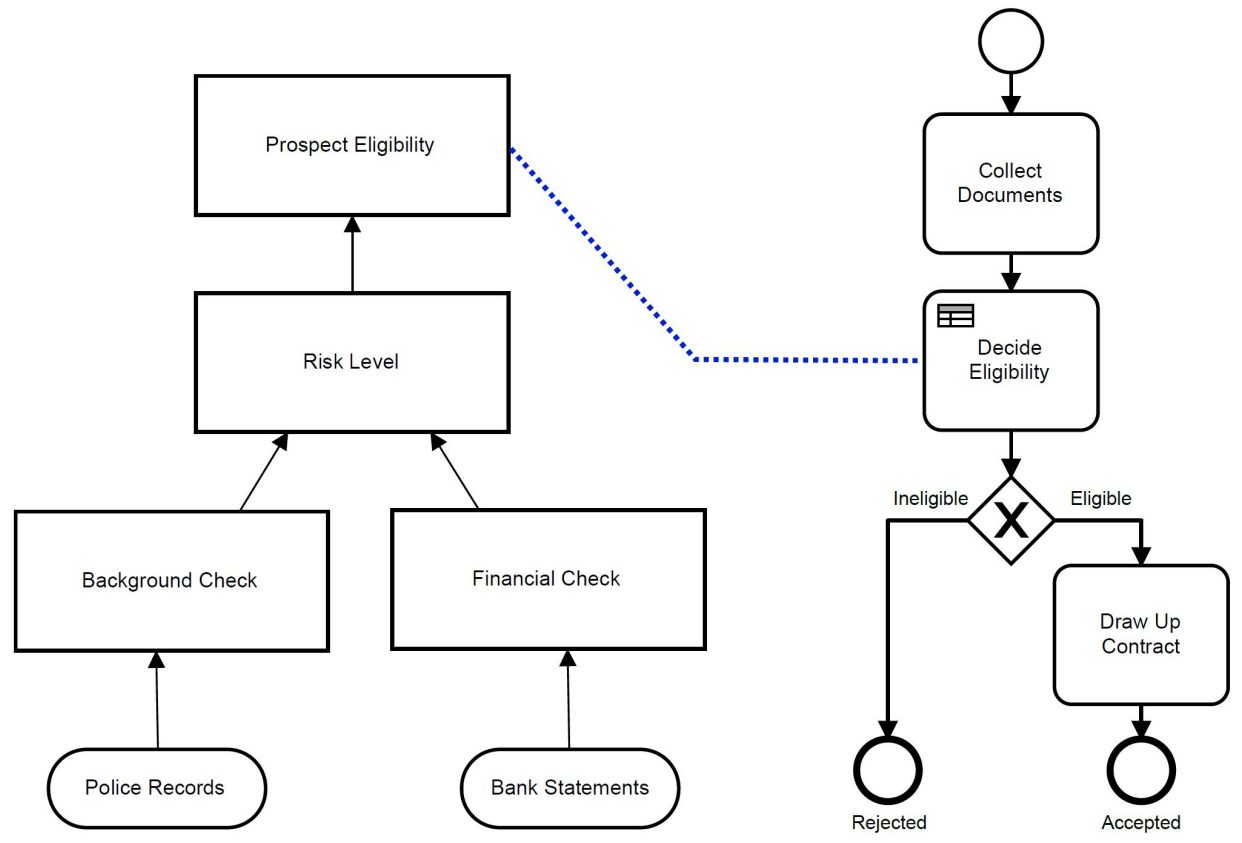

Figure 1: Prospect acceptance decision model linked to a process model.

e-service, thus, providing citizens with information and transparency in the area of income tax regulations.

This paper is structured as follows. In Section 2 , relevant works on decision modelling are provided. Section 3 provides modelling strategies for the decision logic layer of DMN, while Section 4 outlines a modelling strategy for the construction of DMN decision models in general. In Section 5 , the case of tax decision management for travelling artists is elaborated upon, and the government e-service is built according to the proposed modelling strategies. Subsequently, the service is deployed and in Section 6, a discussion and evaluation of the case study is presented. Finally, Section 7 concludes the paper.

\section{Related work and motivation}

In this section, we provide relevant work on decision services and integrated process and decision modelling from the process and decision management field to motivate this paper. Decision modelling refers to developing decision models that encapsulate business logic. These decision models can then be integrated into processes or other systems. A plethora of authors 
have suggested to approach decision models as a service, hence, obtaining decision services. This way, the logic in the decision model can be queried by multiple business processes, systems, and applications instead of being embedded in a single system.

\subsection{Decision modelling}

The integrated modelling of processes and decisions offer firm motivation for keeping multi-perspective modelling tasks isolated and founded on a basis which can be used to ensure consistency between the models. This integrated modelling was already considered in terms of business rules within processes (Goedertier \& Vanthienen, 2006, Wei et al., 2017). With DMN, integration of decisions and processes has become a possibility, since decisions can be encapsulated in separate decision models and linked to the invoking context, e.g. a business process. This externalisation is inspired by the commonly known separation of declarative models and reasoning techniques in knowledge-based systems Russell \& Norvig (2016). Consequently, more attention was recently given to the integration of process and decision logic (Hasić et al., 2017, Biard et al., 2015; Hasić et al., 2017c) as to illustrate that the DMN standard can play a vital role for knowledge-intensive processes (Santoro \& Baião, 2017) and for business process compliance (Hashmi et al., 2018).

\subsection{Decision services}

Recent business process management (BPM) literature moves towards accommodating decision management into the paradigms of Separation of Concerns (SoC) (Campos et al., 2017, Hasić et al., 2018b) and ServiceOriented Architecture (SOA) (Hasić et al. 2017a), by externalising decisions and encapsulating them into separate decision models. Hence, the decisions are implemented as externalised services. Literature proposes several conceptual decision service platforms and frameworks (Zarghami et al. 2012 Mircea et al., 2011; Hasić et al., 2017a) as industry has adopted this trend. This externalisation of decisions from processes provides a plethora of advantages regarding maintainability and flexibility of both process and decision models (Hasić et al. 2017a; Figl et al., 2018; Hasić et al., 2017; Hu et al., 2017; Hasić et al. 2018b, 2017). However, in order to integrate models, the models have to be developed first. There exist no guidelines and strategies in literature that focus on developing DMN models. That is a missing link which we address in this paper. Furthermore, externalising decision services from processes and applications stimulates their reuse in other processes and systems. That way, other processes and systems can 
invoke the decision model as a service if the enactment of the modelled decision logic is required.

However, despite the fact that strategies and methodologies for the integration of decision and process models have already been studied in literature, scientific literature on strategies for DMN decision modelling are still lacking. This paper aims at approaching that research gap in the following sections and at providing strategies for DMN modelling, both on the logic and the decision requirement level in order to facilitate the creation of e-government services (West, 2004, Carter \& Bélanger, 2005, Rana et al., 2015; Simonofski et al., 2017; Panopoulou et al., 2014). The modelling strategies are subsequently illustrated through a real-life case on an income tax e-service.

\section{DMN logic modelling}

The history of decision modelling and DMN finds its origin in decision tables, where rules for decision logic are represented in a structure of related tables which map combinations of inputs to outcomes. Decision tables and the accompanying methodology have proven a powerful vehicle for acquiring the decision knowledge and for checking completeness, correctness, and consistency (CODASYL Decision Table Task Group, 1982).

DMN builds upon these concepts and goes even further by standardising existing decision table formats (using a hit policy indicator), by elaborating the requirements diagram, and by introducing a standard expression language. Even though DMN standardises and extends the modelling capabilities of decision requirements and decision logic (e.g. by adding FEEL), a lot of knowledge is already available that can be relevant to DMN. We rely on these legacy decision table modelling strategies and guidelines to render a set of guidelines that is applicable to the DMN standard. In what follows, we group the relevant methods and guidelines and we amend and expand them to be applicable to DMN. Wherever we use methods and guidelines from literature, we provide the relevant works.

\subsection{Decision table modelling strategies}

Decision tables can be modelled according to different strategies, depending on what is available at the start of the modelling process:

\subsubsection{Direct method}

Very often, decision tables are built based on an available description in the form of a text, procedure, law. This specification is basically the 
only available source to start the modelling process, although a domain expert might be available in case of questions that turned up during the modelling process. In the direct method for constructing decision tables, the following modelling steps can be distinguished, as described in (Merlevede \& Vanthienen, 1991):

1. Obtain conditions, condition intervals, and outcomes of the decision situation.

2. Specify the problem in terms of decision rules.

3. Fill the decision table based on the rules.

4. Check for completeness, correctness, and contradictions.

5. Simplify the decision table and display it.

Note that step four can happen automatically, as a number of tools have been developed to perform these checks, e.g. Calvanese et al. (2018).

\subsubsection{Interactive method}

Here the decision tables are built in close interaction with the domain expert. In a dialogue mode, the modeller and the expert gradually discover relevant criteria and outcomes and refine the table until a full description of the decision logic is obtained.

\subsubsection{Mining method}

Decision logic can also be derived from case data where the mined model is discovered or transformed into a decision table (Wets et al., 1998; Baesens et al. 2003). Not unlike process models, which can be discovered from events logs, decision discovery is a form of knowledge discovery from logs containing historical data about case attributes and their outcome. Currently, decision mining is often limited to discovering the decision logic at a certain decision point in a process model, but a more complex challenge is the integrated mining of both a process and a decision model based on extensive decision process $\operatorname{logs}$ (De Smedt et al., 2016, 2017; De Smedt et al., 2019; Campos et al. 2017).

Notice that the input data of a decision table corresponds to the preconditions modelled during decision table construction. In the mining method the input data are automatically discovered from data patterns in data logs, in the direct method the input data are obtained in the first step, and in the interactive method they are discovered interactively and iteratively in a dialogue with the domain experts. 


\subsection{Decision table modelling guidelines}

Although DMN is mainly about notation, and is not meant to include a design methodology, there is a long history of decision table design guidelines, offering guidance to structure decisions into separate tables, to build sound decision tables using a step-wise methodology and to avoid table anomalies and non-normalised tables (Vanthienen \& Snoeck, 1993). These guidelines deal with the form as well as with the contents of the table and emanate from the need to use the decision table as a well structured tool across various application areas (Vanthienen \& Snoeck, 1992).

\subsubsection{Structure and content}

1. Basic structure: Decision tables represent rules about related conditions and outcomes. The purpose of the decision table is that a given combination of condition entries leads to a specific set of outcomes. By definition of the decision table, all elements in one rule (condition and outcome combinations) are implicitly connected with AND, all rules are connected with OR.

2. Completeness: There should be no missing combinations. But completeness can be obtained in two ways: either by design or by providing a remainder column which catches all missing rules. The latter solution, although complete by definition and even compact, is less elegant and way more difficult to understand, validate, and maintain.

3. Consistency of the rules: The most important question in describing types of decision tables, is the question of overlapping rules. Tables with overlapping rules, even if these rules produce the same result, are more difficult to validate manually. Because the rules of the table are not mutually exclusive, there is at least one combination of condition entries that matches two rules. Good decision tables have to meet the demand of completeness and consistency with respect to the rules. Each combination of condition entries should be incorporated in one and only one rule (row or column). Otherwise the table is not complete or produces inconsistent results. Because decision tables are relations, this is simply the requirement of normalisation. Consistency can be obtained in two ways: either by design or by providing a mechanism that resolves overlapping combinations (e.g. the first hit convention to obtain single-hit tables, also known as the first hit policy). Although both designs will produce a consistent table, the latter is more difficult to verify by humans and requires tool support to ensure consistency. Verification and validation of rule-based systems (including decision tables) has been a major area of research, as exemplified by 
the earlier EUROVAV series of conferences (European Conference on Verification \& Validation of Knowledge-based systems) (Antoniou et al., 1998). There are numerous works dealing with $\mathrm{V} \& \mathrm{~V}$ of a set of rules (as present in single decision tables). Typical rule anomalies are: redundancy (including duplicates and subsumption), ambivalence, circularity, and deficiency (missing rules). Numerous algorithms are available for checking and eliminating contradictions, redundancies and missing rules for all possible values of the input variables. For an overview of earlier research in this area, see (Vanthienen et al. 1998). Other approaches have been designed to strictly avoid table anomalies by recommending unique single hit tables.

4. Consistency over multiple decision tables: Verification and validation of table structures has been covered in earlier research. When input conditions or outcomes are repeated in more than one decision, some parts of the decision logic in a certain decision may become unreachable or inconsistent for specific input values. Checking consistency and completeness between interconnected decision tables, i.e. over rule chains, is a much more challenging problem than verification of single tables. See (Vanthienen et al., 1997) for an overview of inter-tabular verification.

5. Refined outcomes: Decision tables can have multiple outcomes. If the purpose of the table is to assign an outcome to a (sub)decision, the main action will assign that outcome, e.g. true/false, classification results, values. There may also be additional outcomes, depending on the purpose of the table.

\subsubsection{Form, conciseness and readability}

1. Group oriented contraction: In the expanded decision table, all combinations of condition values are explicitly enumerated, while in the contracted decision table, adjacent columns or groups of columns that only differ in the entry for one condition and that contain the same outcome configuration are joined, thus, reducing the number of columns. When all entries of a condition can be joined, the entry - (irrelevant) appears in the table. Contraction minimises the number of columns for a given condition order. If all entries for a condition are - throughout the entire table, the condition carries no relevant entries and is therefore redundant. It can simply be removed because the actions do not depend on this condition (normalisation rule).

2. Row order optimisation: Row order optimisation determines the condition order which results in the minimum number of columns. In a decision table, the condition order is the same for all columns of the table, but a different overall condition order may produce a smaller table because of contraction. For a table with $n$ conditions, this implies a choice between $n$ ! 
alternative condition orders.

3. Optimal execution: When properly specified using (S)FEEL, decision models and tables are executable. This is a straightforward execution without further optimisation. In a number of cases, however, e.g. when the test times of conditions are very diverse or when some rules will be executed much more frequently than others, attention can be paid to execution efficiency or more flexible forms of execution. Since the early days of decision tables, a lot of work has been done in this area, by transforming decision tables into optimal test sequences, by generating least-cost execution trees based on condition test times and case frequencies, see e.g. (Lew, 1978, CODASYL Decision Table Task Group, 1982).

\subsubsection{Factoring and normalisation}

1. Normalisation: Because decision tables are relations between condition entries and outcomes, normalisation theory can be used to obtain sound decision tables. Normalisation of decision tables (Vanthienen \& Dries, 1994) allows to simplify and split up tables into more clean structures. Decision tables can (or should) be split up if the outcomes are not dependent on all the conditions. This is called factoring or normalisation, analogous to normalisation in relational database theory where attributes should be dependent on the key, the whole key and nothing but the key.

2. Subtables: Most decision problems are too large to fit in one decision table and are divided into problem segments which are then analysed separately. A decision table invokes another table by having the subtable returning a value which is needed by the invoking condition. This is also what is expressed in the DMN requirements diagram where a decision has an information requirement from another decision. Chapter 5 of the CODASYL report (CODASYL Decision Table Task Group, 1982) already contains an elaborate example of a problem hierarchy with subtables.

\subsubsection{Purpose}

1. Selection structure (no initialisation or repeat actions): A decision table is the representation of a complex multiple selection. It should therefore not include initialisation or iteration facilities in the table itself. These features can be realised using the proper surrounding features or DMN constructs. Moreover, as condition subtables are considered equivalent to functions, a table referring to itself would be regarded as a recursive table. 


\section{DMN requirements modelling strategies}

In the previous sections, we have discussed modelling methodologies and guidelines for the logic level of the DMN standard, i.e., the decision tables. In this section, we address modelling strategies for DMN in general, i.e., including the decision requirements hierarchy as depicted in the decision requirements graph (DRD), while bearing in mind the guidelines and strategies for logic modelling as elaborated upon in Section 3. This section outlines possible approaches towards building decision models and discusses which approaches appear in which scenarios. The strategies are based upon wellestablished modelling approaches in the BPM community (Verner, 2004, Lin et al., 2002), i.e., bottom-up, top-down, and combined modelling concepts, adapted to suit DMN modelling.

\subsection{Decision requirements first, before decision logic}

This top-down approach corresponds with a goal-oriented view of decisions: first the target decision or top-level decision is identified and subsequently, the top-level decision is decomposed into lower-level decisions, thus, transforming the decision model into a finer grained model with every decomposition. After determining the decision hierarchy through these decomposition iterations, the underlying decision logic is modelled as well and, if necessary, the decision model is annotated with additional elements. This top-down approach is modelled through the following steps:

1. Determine the top-level decision.

2. Decompose the top-level decision into subdecisions.

3. Decompose the subdecisions further into lower-grained decisions.

4. Model the decision hierarchy.

5. Model all relevant inputs, knowledge sources, and knowledge models.

6. Model the underlying decision logic.

7. Test the model and, if needed, reiterate over the previous steps.

\subsection{Layered approach: Top decisions first, decision logic included}

The layered approach is similar to the previous approach in the sense that both approaches are initialised with the determination of the top-level decision. However, instead of first decomposing the top-level decision to 
obtain the decision hierarchy like in the top-down approach, the layered approach first models the underlying decision logic of the top-level decision. In this approach, the decomposition into lower-level decisions goes together with the construction of the decision tables for the lower-level decisions. That way the decision hierarchy is built from the top of the decision model towards the bottom. Once the decision hierarchy is known, the model can be expanded and relevant data and knowledge elements can be added. The layered approach contains the following steps:

1. Determine the top-level decision.

2. Model the underlying decision logic of the top-level decision.

3. Decompose the top-level decision into subdecisions and construct the corresponding decision tables.

4. Continue this decomposition with the newly formed lower-level decisions until a satisfactory level of decision granularity is reached.

5. Model all remaining input data, knowledge sources, and knowledge models.

6. Test the model and, if needed, reiterate over the previous steps.

\subsection{Bottom-up approaches}

Unlike the previous two approaches, the bottom-up approach does not start with the top-level decision. Rather, any decisions that can be identified from data or text, e.g. certain regulations or laws, can be modelled as a decision with a corresponding decision table. Afterwards, the relevant decisions and decision tables can be linked with each other to form the decision hierarchy leading to the top-level decision. The resulting decision model is then further enriched with data, knowledge sources, and business knowledge models. Note that two kinds of bottom-up approaches are possible: one where the decision hierarchy is modelled first, followed by the detailed modelling of the underlying decision logic, and one where the decisions and decision tables are established together and then linked into higher-level decisions. The bottom-up approach contains the following steps:

1. Determine any decisions and the corresponding decision tables that can be extracted from data or from documentation.

2. After determining a number of decisions and decision tables, try to link them in order to establish a decision hierarchy. 
3. Link the decision hierarchy until a top-level decision is identified.

4. Model all remaining input data, knowledge sources, and knowledge models.

5. Test the model and, if needed, reiterate over the previous steps.

\subsection{Combined approaches}

The combined approach is a mix between the bottom-up and the topdown approach. One can identify higher-level decisions and then start to decompose them top-down, while also identifying lower-level decisions and trying to connect them to higher-level decisions. That way, the decision hierarchy can be established using both directions, i.e. top-down and bottom-up. The combined approach can be layered or not, or a combination of a layered and non-layered approach: either the decisions are decomposed first and the logic is added later, or the decision logic is modelled and the decision table is decomposed into subdecisions or decision tables are merged into higher-level decisions. That way, a number of combined strategies are possible:

- Combined top-down and bottom-up approach with decision hierarchy first, before the decision logic is modelled.

- Combined top-down and bottom-up approach with a layered approach where decision logic is modelled first, and the decision hierarchy is established afterwards by linking decision tables, decomposing or merging them.

- Combined top-down and bottom-up approach with both layered and non-layered concepts, i.e. parts of the model are built with decision hierarchy first and afterwards the logic is modelled, while other parts first model decision tables and then merge or decompose them to obtain a decision hierarchy.

\subsection{When to use which approach}

Top-down requirements first approaches are suitable for goal-oriented decision making problems where it is clear what the top-level decision is. That top-level decision is consequently decomposed into decisions with higher granularity. When the underlying decision logic is not clear from the start (or not relevant yet), the emphasis lies on constructing the decision hierarchy first by logically decomposing the decisions. Afterwards, each of the decisions in the decision model is detailed with decision logic to fit the business needs that the top-level decision pertains to. 
Top-down layered approaches are, like top-down approaches, goal-oriented. However, in the layered approach, the decision logic of the top-level decision is already available. The logic of the top-level decision is then modelled in the form of a decision table and the decomposition step of the top-level decision is combined with the construction of decision tables for each lower-level decision. This process continues until a satisfactory level of granularity is reached.

Bottom-up approaches are typical in situations where a lot of documentation and data is available. The goal here is to transform large amounts of complicated texts, e.g. laws, regulations and business rules hidden in text and documentation, into a crisp and clear decision model. This is typically done by identifying rules and decisions in the documentation and by consolidating the rules into decision tables. Afterwards, the decision tables are linked with each other and annotated where needed to form the decision hierarchy.

Combined approaches are likely to occur in most real-life cases. Usually, when aiming to construct a decision model, it is clear what the top-level decision is the organisation is aiming for. On the other hand laws, regulations and business rules are available in the data and documentation present in the organisation. That way both lower-level decisions are identified in the documentation and the relevant business rules are transformed into a decision table format. the decision hierarchy is consequently established in both directions, i.e. bottom-up and top-down. The known top-level decision can be decomposed towards the modelled lower-level decisions and decision tables obtained from documentation. Likewise, the lower-level decisions and decision tables can be linked or even merged into higher-level decisions and decision tables, thus, moving towards the top-level decision.

\section{Advanced tax management for travelling artists}

In this section, we address a real-life complex decision management problem of tax regulations for visiting performing artists in Belgium. First, we provide a problem statement, followed by an argumentation of the followed modelling strategies and methodologies for the problem at hand. Finally, we discuss the results in terms of the model that was built in the Avola decision management tool as well as the implementation of the resulting decision model as an e-service. 


\subsection{Problem statement}

Like researchers, artists often travel to other countries to present their work. Often arts and culture societies would invite foreign performing artist to showcase their latest creation: e.g. a theatre play or an opera piece. The artists have to be remunerated for their effort in the country where they perform. With remuneration, questions regarding income taxation arise as well. Will the artists be taxed in Belgium, i.e., the country where they are invited to perform, or in their home country? Will they be subject to double taxation, i.e., both in their originating country and in the country of the performance? Are the artists eligible for tax reductions in the performing country, or even full tax exemptions? The legislation on income tax for these specific situations is quite complex, and so are the double taxation agreements between different countries, as they all tend to differ from each other quite significantly. Hence, there is a need for simplification and transparency, not only for the artists who need to know how much they can earn, where to file for taxes and under which tariffs, but also for arts and culture institutions inviting foreign artists to their country, production studios invoking the help of foreign artists in their productions, and even government employees charged with tax collection. Given the complexity of the problem at hand, a case by case evaluation is necessary to make the correct decision. This is a rather burdensome and unstructured processes. Using the modelling strategies and methodologies described in the previous sections, a decision model can be built that automatically evaluates the cases of artists in an unambiguous manner. This decision model can be offered as a service to all parties involved in the tax management problem: government agencies, artists subject to taxation, production studios, and arts and culture institutions and federations.

\subsection{Applied modelling strategies}

In this subsection, we provide an argumentation of the followed modelling strategies for the problem described in the previous subsection. We address the approaches followed for decision logic modelling as well as DMN modelling in general.

\subsubsection{Decision logic modelling}

The problem described in Section 5.1 is in essence a legal problem as the information relevant for the tax decision is available in the form of law texts

and procedures. Hence, the primary decision logic modelling strategy that needs to be applied here is the direct method as described in Section 3.1.1. 
In the direct method the construction of decision tables is performed by obtaining conditions, relevant condition intervals and outcomes of the decision situation from the textual descriptions. These textual descriptions are then transformed into a more formal representation in the form of decision rules. This leads to a set of tax rules obtained from the legal descriptions. These tax rules are consequently logically bundled in decision tables. The decision tables can then be checked for completeness, correctness and possible contradictions. After this post processing step, the decision tables are simplified and represented according to the decision table modelling guidelines described in Section 3.2 to ensure their structure, content, form, conciseness, readability, factoring, purpose, and possible indications of impossibilities.

However, the direct method was not sufficient to model the tax management problem, as the law texts are at times rather complex to understand without the necessary legal training. The modellers have an information systems background and not a legal one. Therefore, a secondary strategy was used as well: the interactive method as described in 3.1.2. This interactive method allows to build tables in close interaction with domain experts. In a dialogue mode, the modeller and the experts gradually discover relevant criteria and outcomes and refine the tables until a full description of the decision logic is obtained and the legal descriptions are understood and made unambiguous. For this tax management problem, we had six iterative meetings to validate, amend, clarify, and improve the decision logic obtained in the modelling cycles from the law texts through the direct method. The knowledge authorities consulted in the interactive method are legal experts attached to either a Belgian industry federation for performing artists or the ministry of culture and media. The experts in both industry and government provided valuable input towards understanding all legal requirements specified in laws, tax agreements, and government policies. This ensured to correct translation of legal requirements into decision rules.

\subsubsection{Decision requirements modelling}

The strategy used for modelling the DMN tax management model in general is rather eclectic and complex, as it combines a top-down approach with a bottom-up approach together with principles of a layered approach. This approach was also briefly described in Section 4.4. For the problem at hand it is clear what the top-level decision is, i.e., the tax policy that will be applied to the income of the foreign visiting performing artist. Hence, a top-down approach is used to further decompose the top-level decision in order to obtain the decision hierarchy. On the other hand, rules in the form of law and regulations are available in a textual format and relevant 
decisions for income tax on foreign artists can be identified in the documentation and transformed into a decision table format. This corresponds to a bottom-up approach. Note that according to the layered approach the decision logic is modelled first based on the rules derived from legal texts, before the actual decision hierarchy is constructed. The decision hierarchy is consequently established in both directions, i.e. bottom-up and top-down: The decision tables obtained for the identified decisions can be linked, merged or decomposed in order to obtain a decision requirement graph. Thus, the modelling strategy is a combination of a bottom-up and top-down approach where the decision logic is obtained first, before the construction of the decision hierarchy, i.e. a layered approach. Similar to the approach followed for decision logic modelling, the decision hierarchy was discussed with the domain experts from the industry federation and the government institution. This DMN modelling endeavour went through a handful of cycles before consensus was reached among the modellers and all the experts.

\subsection{Resulting decision model}

The modelling strategies, as explained in Section 5.2, applied during the modelling cycles of the tax management problem resulted in a DMN model that distinguishes eight core decisions. A requirements representation of the decision model can be found in Figure 2. Additionally, a more detailed view of the top-level decision Income Tax is provided in the form of a decision table in Figure 3. Note that this table, contrary to Guideline 3 in Section 3.2.1, is a first hit table. This is due to the fact that the Avola decision modeller only accepts first hit policy tables. Next, we explain the relevant decision nodes of the decision model:

- Income Tax is the top-level decision and it provides the category of income tax that the travelling artist belongs to, e.g. taxed in country of labour, not taxable in country of labour, the necessity to fill in additional forms (e.g. if not all performing artists come from the same country, each of them will have to fill in the form separately).

- Subsidised Exemption determines whether the artist can enjoy tax exemptions based on subsidies allocated to the artist's organisation by the government.

- Individual Exemption investigates whether the artist can enjoy some tax exemptions based on his personal situation, e.g. depending on the number of full time equivalent days that he worked in the country of the performance. 
- Organisational Exemption determines whether the artist can enjoy tax exemptions based on potential special treatments and agreements that the artist's working organisation enjoys from the government of the country of performance.

- Fiscal Articles figures out which fiscal articles are applicable to the artist.

- Double Tax Agreement indicates the logic and the rates of the double tax agreement between the originating country and the country of performance.

- Initiation of Double Tax Agreement gives the date of validity of the double tax agreement between the originating country and the country of performance.

- Application Area evaluates whether the time the artist worked in the country he visited falls under a period of double tax agreements between the artist's country of origin and the country of performance.

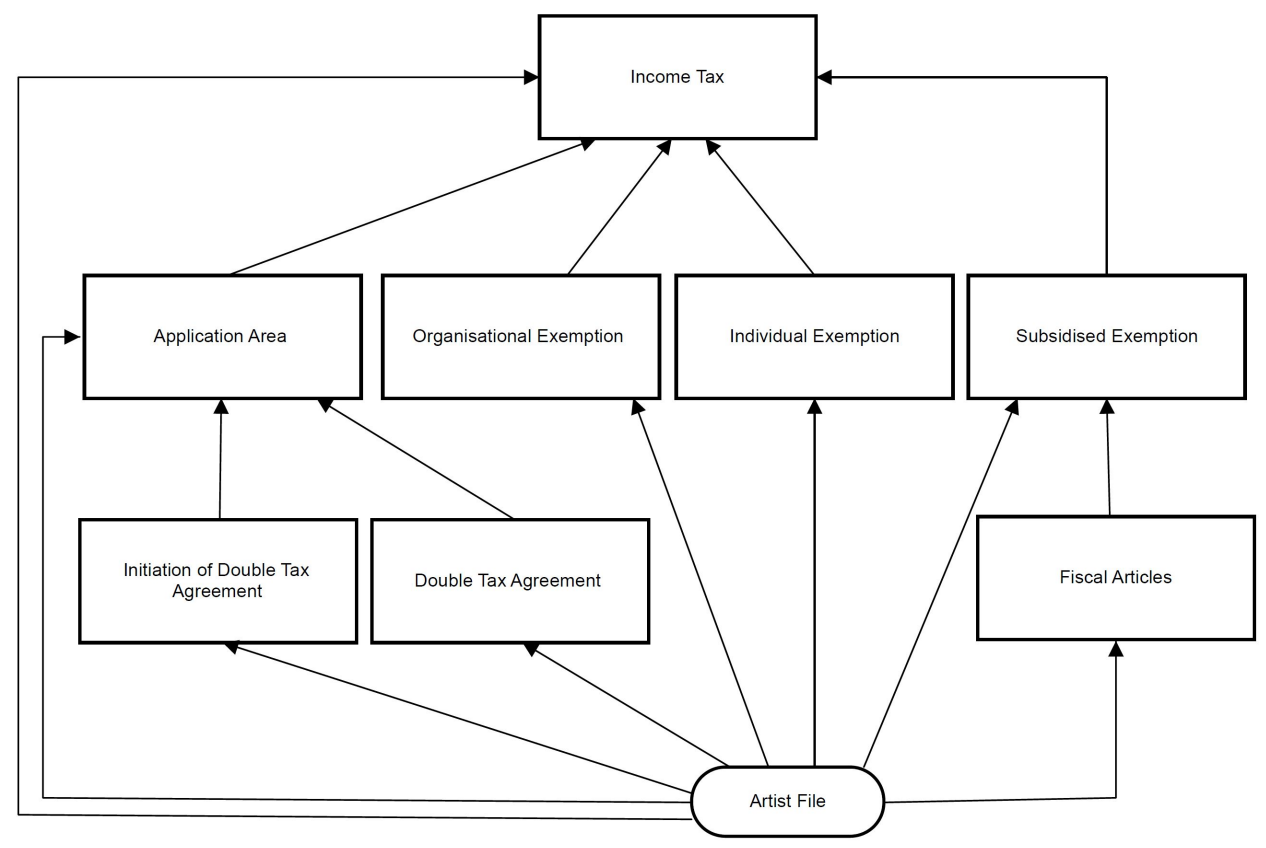

Figure 2: Tax management decision model. 


\begin{tabular}{|c|c|c|c|c|c|}
\hline Application Area & $\begin{array}{c}\text { Artist Same } \\
\text { Home Country }\end{array}$ & $\begin{array}{l}\text { Individual } \\
\text { Exemption }\end{array}$ & $\begin{array}{c}\text { Organisational } \\
\text { Exemption }\end{array}$ & $\begin{array}{l}\text { Subsidised } \\
\text { Exemption }\end{array}$ & Income Tax \\
\hline $\begin{array}{l}\text { Double tax } \\
\text { agreement not } \\
\text { implemented }\end{array}$ & & & & & Fill in paper form \\
\hline $\begin{array}{c}\text { No double tax } \\
\text { agreement }\end{array}$ & & & & & $\begin{array}{c}\text { Taxed in country of } \\
\text { labour }\end{array}$ \\
\hline $\begin{array}{c}\text { Double tax } \\
\text { agreement valid }\end{array}$ & $\begin{array}{c}\text { Different home } \\
\text { countries }\end{array}$ & & & & $\begin{array}{c}\text { Fill in separate form } \\
\text { per nationality }\end{array}$ \\
\hline $\begin{array}{c}\text { Double tax } \\
\text { agreement valid }\end{array}$ & $\begin{array}{l}\text { Same home } \\
\text { country }\end{array}$ & $\begin{array}{c}\text { Exemption not } \\
\text { possible }\end{array}$ & & & $\begin{array}{c}\text { Taxed in country of } \\
\text { labour }\end{array}$ \\
\hline $\begin{array}{c}\text { Double tax } \\
\text { agreement valid }\end{array}$ & $\begin{array}{l}\text { Same home } \\
\text { country }\end{array}$ & $\begin{array}{l}\text { Exemption } \\
\text { possible }\end{array}$ & $\begin{array}{l}\text { Exemption } \\
\text { possible }\end{array}$ & & $\begin{array}{c}\text { Exempt of income } \\
\text { taxation in country of } \\
\text { labour }\end{array}$ \\
\hline $\begin{array}{c}\text { Double tax } \\
\text { agreement valid }\end{array}$ & $\begin{array}{c}\text { Same home } \\
\text { country }\end{array}$ & $\begin{array}{c}\text { Exemption } \\
\text { possible }\end{array}$ & $\begin{array}{c}\text { Exemption not } \\
\text { possible }\end{array}$ & & $\begin{array}{c}\text { Taxed in country of } \\
\text { labour }\end{array}$ \\
\hline $\begin{array}{l}\text { Double tax } \\
\text { agreement valid }\end{array}$ & $\begin{array}{l}\text { Same home } \\
\text { country }\end{array}$ & & & $\begin{array}{l}\text { Exemption } \\
\text { possible }\end{array}$ & $\begin{array}{c}\text { Exempt of income } \\
\text { taxation in country of } \\
\text { labour }\end{array}$ \\
\hline
\end{tabular}

Figure 3: Income tax first hit decision table.

\subsection{E-service deployment}

Based on the decision model that was built, a decision e-service was developed that returns the decision outcome of the top-level decision Income Tax given a number of input data entries provided by the user of the service. The Avola decision management tool allows to easily deploy an e-service of the decision model that was developed in the tool. This service can be used by artists to query in which income tax category they belong, by industry organisations and production houses that wish to employ foreign performing artists, as well as by government employees for decision support in tax collection. That way, tax rule compliance becomes transparent to all parties and ambiguities are circumvented. Figure 4 gives an overview of the necessary input data needed to invoke the decision model through the eservice. Note that for the time being the service was only set up for artists visiting Belgium and that the service input is in Dutch. We briefly sum up the data input fields needed for the service invocation: the country in which the artist is performing (currently only implemented for Belgium), the origin country of the performing artist, type of legal personality of the organisation or artist, the origin country of the performing organisation, income earned by the artist in the visiting country in the past year, whether all performing artists of the performance share the same origin country within the organisation, date of performance, whether the artist worked for more than 183 
days over the past year in the country that he is visiting, whether the artist is eligible for personal exemption, and the percentage of government subsidies allocated to the performance. By providing these ten input values, the e-service invokes its underlying decision model and returns the tax policy category.

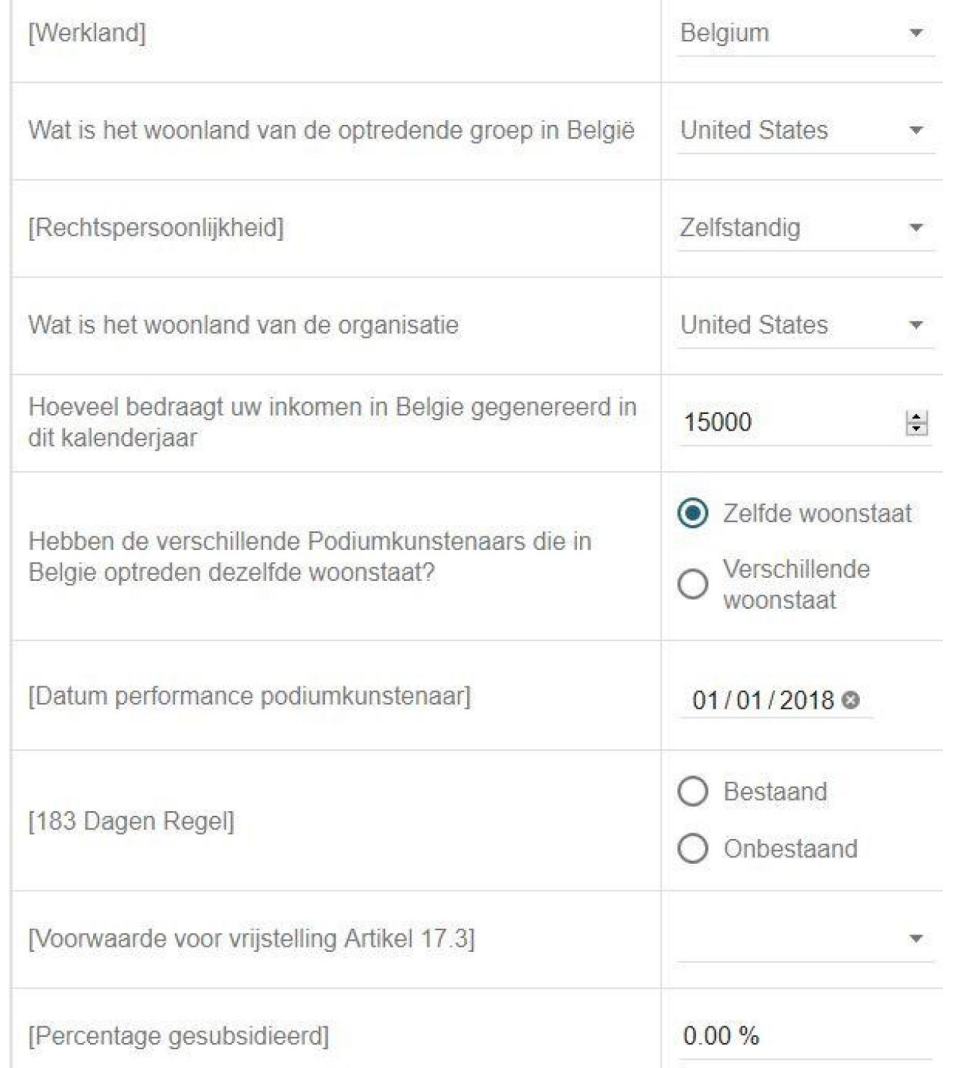

Figure 4: E-service input requirements for the tax management decision model (in Dutch).

\section{Discussion}

The modelling process elucidated in Section 5 took approximately two months consisting of six rounds of validation with two domain experts, one from the industry federation and one linked to the Ministry of Culture. An initial model was extracted from law texts which was provided to us by the domain experts. In the subsequent rounds of meetings with the experts, the 
model was amended and validated iteratively. Noteworthy is that on questions regarding specific scenarios, the experts themselves had difficulties to provide a clear answer. This is due to the complexity of taxation regulations, as the experts had to consult a plethora of legal sources to answer convoluted questions. However, in the more advanced rounds of the modelling processes the decision logic was more and more gathered and centralised in a set of decision tables of one decision model. This provided the experts themselves with a better overview of the legal rules and they were able to discuss more convoluted and advanced scenarios with more certainty. Thus, the modelling strategies aid in centralising and structuring the decision information. After the experts agreed on the final model, they devised five testing scenarios to check whether the model would provide the correct answer. They tested both simple and more convoluted situation and concluded that the model reflects the actual rules of the taxation regulation. This was the expected outcome of the tests as the experts were mainly concerned with the correct translation of the legal prescriptions into decision logic during the modelling process. The e-service has been implemented inside the industry federation and is available for employees of the industry federation, not for the artists themselves. The service provides decision support to the employees and hence makes that the tax computation process is quicker and more transparent. Previously, all special scenarios had to be examined by the designated expert. This delayed the processes of tax calculation and tax exemption.

In analogy with the model constructed for Belgium in this paper, the service can be expanded for other countries of the European Union and offered in different languages. Currently, the Belgian industry federation for the arts, oKo, is looking towards sharing this model with the European industry federation which will then disseminate the approach to all national and regional industry federations in order to achieve unanimity in tax transparency across the European Union.

\section{Conclusion}

In this paper, we provided an approach for the creation of government e-services based on decision modelling according to the Decision Model and Notation (DMN) standard. The DMN standard provides opportunities for rapid and sound e-service creation for governments, given the standard's ease of use and comprehensibility. Thus, laws and regulations can be transformed into DMN models which can subsequently be deployed as e-services, 
thus, providing citizens with information and transparency in areas of government regulations. More specifically, in this paper, we provided modelling strategies for DMN both at the decision requirements level and at the decision logic level. We discussed which modelling strategy is most suitable in certain scenarios and we provided limitations of the proposed strategies, as well as opportunities provided by the strategies. Additionally, a real-life decision model on the problem of tax regulations for visiting performing artists in Belgium was built in order to illustrate how an e-service can be obtained through DMN by adhering to the proposed modelling strategies and guidelines provided in this paper.

In future work, we will further investigate how citizen participation can aid in the effective development and deployment of e-services based on DMN decision models. Furthermore, we will work out additional e-services by capitalising on the DMN standard and we will test those services for user friendliness.

\section{Acknowledgements}

We would like to thank Avola for giving us access to their tool. Furthermore, our thanks to the Belgian industry federation for the arts (oKo) and Cultuurloket (of the Ministry of Culture) for their support on the interpretation of the laws.

\section{References}

Antoniou, G., van Harmelen, F., Plant, R., \& Vanthienen, J. (1998). Verification and validation of knowledge-based systems: Report on two 1997 events. AI Magazine, 19, 123.

Baesens, B., Setiono, R., Mues, C., \& Vanthienen, J. (2003). Using neural network rule extraction and decision tables for credit-risk evaluation. Management science, 49, 312-329.

Biard, T., Le Mauff, A., Bigand, M., \& Bourey, J.-P. (2015). Separation of decision modeling from business process modeling using new decision model and notation(dmn) for automating operational decision-making. In Working Conference on Virtual Enterprises (pp. 489-496). Springer.

Calvanese, D., Dumas, M., Laurson, Ü., Maggi, F. M., Montali, M., \& Teinemaa, I. (2018). Semantics, analysis and simplification of dmn decision tables. Information Systems, 78, 112-125. 
Campos, J., Richetti, P., Baião, F. A., \& Santoro, F. M. (2017). Discovering business rules in knowledge-intensive processes through decision mining: an experimental study. In International Conference on Business Process Management (pp. 556-567). Springer.

Carter, L., \& Bélanger, F. (2005). The utilization of e-government services: citizen trust, innovation and acceptance factors. Information systems journal, 15, 5-25.

CODASYL Decision Table Task Group (1982). A modern appraisal of decision tables. Association for Computing Machinery.

De Smedt, J., vanden Broucke, S. K., Obregon, J., Aekyung, K., Jung, J.-Y., \& Vanthienen, J. (2016). Decision mining in a broader context: an overview of the current landscape and future directions. In Business Process Management Workshops Lecture Notes in Business Information Processing. Springer.

De Smedt, J., Hasić, F., vanden Broucke, S. K., \& Vanthienen, J. (2019). Holistic discovery of decision models from process execution data. Knowledge-Based Systems, 183, 104866.

De Smedt, J., Hasić, F., \& Vanthienen, J. (2017). Towards a holistic discovery of decisions in process-aware information systems. In Business Process Management Lecture Notes in Business Information Processing. Springer.

Deryck, M., Hasić, F., Vanthienen, J., \& Vennekens, J. (2018). A case-based inquiry into the decision model and notation $(\mathrm{dmn})$ and the knowledge base (kb) paradigm. In International Joint Conference on Rules and Reasoning (pp. 248-263). Springer.

Figl, K., Mendling, J., Tokdemir, G., \& Vanthienen, J. (2018). What we know and what we do not know about dmn. Enterprise Modelling and Information Systems Architectures, .

Ghlala, R., Aouina, Z. K., \& Said, L. B. (2017). Mc-dmn: Meeting mcdm with dmn involving multi-criteria decision-making in business process. In International Conference on Computational Science and Its Applications (pp. 3-16). Springer.

Goedertier, S., \& Vanthienen, J. (2006). Compliant and flexible business processes with business rules. In Proceedings of the CAISE Workshop on Business Process Modelling, Development, and Support BPMDS. 
Hashmi, M., Governatori, G., Lam, H.-P., \& Wynn, M. T. (2018). Are we done with business process compliance: state of the art and challenges ahead. Knowledge and Information Systems, (pp. 1-55).

Hasić, F., De Craemer, A., Hegge, T., Magala, G., \& Vanthienen, J. (2018a). Measuring the complexity of dmn decision models. In International Conference on Business Process Management (pp. 514-526). Springer.

Hasić, F., De Smedt, J., \& Vanthienen, J. (2017). An Illustration of Five Principles for Integrated Process and Decision Modelling (5PDM). Technical Report KU Leuven.

Hasić, F., De Smedt, J., \& Vanthienen, J. (2017). Developing a modelling and mining framework for integrated processes and decisions. In OTM Confederated International Conferences "On the Move to Meaningful Internet Systems". OTM Workshops (pp. 259-269). Springer volume 10697 of Lecture Notes in Computer Science.

Hasić, F., De Smedt, J., \& Vanthienen, J. (2017a). A service-oriented architecture design of decision-aware information systems: Decision as a service. In On the Move to Meaningful Internet Systems Lecture Notes in Computer Science. Springer.

Hasić, F., De Smedt, J., \& Vanthienen, J. (2017b). Towards assessing the theoretical complexity of the decision model and notation (dmn). In Enterprise, Business-Process and Information Systems Modeling Springer International Publishing.

Hasić, F., De Smedt, J., \& Vanthienen, J. (2018b). Augmenting processes with decision intelligence: Principles for integrated modelling. Decision Support Systems, 107, $1-12$.

Hasić, F., Devadder, L., Dochez, M., Hanot, J., De Smedt, J., \& Vanthienen, J. (2017c). Challenges in refactoring processes to include decision modelling. In Business Process Management Workshops LNBIP. Springer.

Hasić, F., \& Vanthienen, J. (2019). Complexity metrics for dmn decision models. Computer Standards \& Interfaces, . URL: http:// www.sciencedirect.com/science/article/pii/S0920548918303647. doi:https://doi.org/10.1016/j.csi.2019.01.006.

Hasić, F., Vanwijck, L., \& Vanthienen, J. (2017). Integrating processes, cases, and decisions for knowledge-intensive process modelling. In International Workshop on Practicing Open Enterprise Modeling. CEUR. 
Hu, J., Aghakhani, G., Hasić, F., \& Serral, E. (2017). An evaluation framework for design-time context-adaptation of process modelling languages. In Practice of Enterprise Modelling (PoEM) Lecture Notes in Computer Science. Springer.

Lew, A. (1978). Optimal conversion of extended-entry decision tables with general cost criteria. Communications of the ACM, 21, 269-279.

Lin, F.-R., Yang, M.-C., \& Pai, Y.-H. (2002). A generic structure for business process modeling. Business Process Management Journal, 8, 19-41.

Merlevede, P., \& Vanthienen, J. (1991). A structured approach to formalization and validation of knowledge. In Developing and Managing Expert System Programs, 1991., Proceedings of the IEEE/ACM International Conference on (pp. 149-158). IEEE.

Mertens, S., Gailly, F., \& Poels, G. (2017). Towards a decision-aware declarative process modeling language for knowledge-intensive processes. Expert Systems with Applications, 87, 316-334.

Mircea, M., Ghilic-Micu, B., \& Stoica, M. (2011). An agile architecture framework that leverages the strengths of business intelligence, decision management and service orientation. Business Intelligence-Solution for Business Development, .

OMG (2019). Decision Model and Notation 1.2.

Panopoulou, E., Tambouris, E., \& Tarabanis, K. (2014). Success factors in designing eparticipation initiatives. Information and Organization, 24, 195-213.

Rana, N. P., Dwivedi, Y. K., Williams, M. D., \& Weerakkody, V. (2015). Investigating success of an e-government initiative: validation of an integrated is success model. Information Systems Frontiers, 17, 127-142.

Russell, S. J., \& Norvig, P. (2016). Artificial intelligence: a modern approach. Pearson Education Limited.

Santoro, F. M., \& Baião, F. A. (2017). Knowledge-intensive process: A research framework. In International Conference on Business Process Management (pp. 460-468). Springer. 
Simonofski, A., Snoeck, M., Vanderose, B., Crompvoets, J., \& Habra, N. (2017). Reexamining e-participation: systematic literature review on citizen participation in e-government service delivery. In Americas Conference on Information Systems. Association for Information Systems (AIS).

Vanthienen, J., \& Dries, E. (1994). Illustration of a decision table tool for specifying and implementing knowledge based systems. International Journal on Artificial Intelligence Tools, 3, 267-288.

Vanthienen, J., Mues, C., \& Aerts, A. (1998). An illustration of verification and validation in the modelling phase of kbs development. Data $\&$ Knowledge Engineering, 27, 337-352.

Vanthienen, J., Mues, C., \& Wets, G. (1997). Inter-tabular verification in an interactive environment. In EUROVAV 1997 (pp. 155-165).

Vanthienen, J., \& Snoeck, M. (1992). Enhanced Decision Modelling through a Decision Table Engineering Workbench. Technical Report KU Leuven.

Vanthienen, J., \& Snoeck, M. (1993). Knowledge factoring using normalisation theory. In International Symposium on the Management of Industrial and Corporate Knowledge (ISMICK'93), October (pp. 27-28).

Verner, L. (2004). Bpm: the promise and the challenge. Queue, 2, 82.

Wei, W., Indulska, M., \& Sadiq, S. (2017). Guidelines for business rule modeling decisions. Journal of Computer Information Systems, (pp. 111).

West, D. M. (2004). E-government and the transformation of service delivery and citizen attitudes. Public administration review, 64, 15-27.

Wets, G., Vanthienen, J., \& Timmermans, H. (1998). Modelling decision tables from data. In Pacific-Asia Conference on Knowledge Discovery and Data Mining (pp. 412-413). Springer.

Zarghami, A., Sapkota, B., Eslami, M. Z., \& van Sinderen, M. (2012). Decision as a service: Separating decision-making from application process logic. In EDOC (pp. 103-112). IEEE Computer Society. 